Originalien

Psychotherapeut 2021 · 66:147-155 https://doi.org/10.1007/s00278-020-00481-3 Angenommen: 4. Dezember 2020

Online publiziert: 29. Dezember 2020

(c) Der/die Autor(en) 2020

\author{
Juan Valdés-Stauber ${ }^{1,4} \cdot$ Helen Kämmerle ${ }^{2}$ Susanne Bachthaler ${ }^{3}$ \\ 'Zentrum für Psychiatrie Südwürttemberg, Ravensburg, Deutschland \\ ${ }^{2}$ Waldburg-Zeil Rehabilitationsklinik, Bad Wurzach, Deutschland \\ ${ }^{3}$ Abteilung Psychosomatische Medizin Ravensburg, Zentrum für Psychiatrie Südwürttemberg, \\ Ravensburg, Deutschland \\ ${ }^{4}$ Klinik für Psychiatrie und Psychotherapie I, Universität Ulm, Ulm, Deutschland
}

\title{
Psychometrische Eigenschaften des Life Attitude Profile - Revised
}

\section{Prüfung der deutschen Version in einem stationär-psychosomatischen Setting}

\begin{abstract}
„Sinn des Lebens“ ist eine allgemeine philosophische Kategorie, nach der der Verstand eine biografische Ordnung sucht und zugleich herstellt. Sinnsuche ist die Grundlage für das Verstehen als rationales Nachvollziehen durch logische Verknüpfung (Valdés-Stauber 2018). "Sinn" in psychologischer Hinsicht unterscheidet sich hiervon: Hier geht es um Bedeutungen, die einerseits durch die Struktur des menschlichen Lebens vorgegeben und andererseits Ergebnis einer personalen „Sinnkonstruktion“ sind.
\end{abstract}

\section{Einleitung}

Die „Suche nach Sinn“ ist insofern eine existenzielle Aufgabe, als das menschliche Leben nicht fertig vorgegeben, sondern von Offenheit, Freiheit, Verantwortung und Selbstsorge gekennzeichnet ist. Vom Menschen verlangt diese Suche nach Sinn, das eigene Leben zu gestalten, und - um es mit den Worten von Heidegger zu sagen - „eigentlich“ zu sein (Heidegger 2006, S. 232-234) und sich im Dasein einzurichten. Der Verlust von Sinn als Bedeutung, wenn diese Bedeutungen auf einer personalen Ebene nicht mehr herstellbar sind, kann zu Krankheit führen, namentlich zu den von Frankl (1994) genannten „noogenen Störungen“.

Eine Verlagerung dieser „Sinnlosigkeit" auf den Resonanzraum des Leibes ist eine wichtige Annahme der psycho- somatischen Medizin. Eine weitere Perspektive stammt aus dem Ansatz der objektiven Hermeneutik, nach der der Sinn aus der Herausarbeitung des Allgemeinen im Einzelfall hervorgeht, durch die das Individuelle Sinn erhält im Abgleich mit dem Allgemeinen (Overmann 2000).

Bei der Dialektik zwischen dem Allgemeinen und dem Individuellen handelt es sich um einen anthropologischen Sachverhalt: eine Dialektik zwischen inhärenten Sinnkategorien der menschlichen Natur und individuellen Sinnkonstruktionen. Diese Sinnkonstruktionen sind personaler Natur und spiegeln, im Sinne Kants ausgedrückt (Kant 1999, S. 130-195), die transzendentalen Sinnkategorien wider, die in einer Person verankert und in ganz besonderer Weise realisiert sind. Darin sind sowohl die Möglichkeiten des Verstehens wie auch des Nichtverstehens begründet. Diese Dichotomie besteht in der semantischen Unterscheidung, wie sie in der englischen Sprache zwischen "meaning" und „significance“ $\mathrm{zu}$ finden ist: Während Significance allgemeinmenschliche geteilte Bedeutungen meint, realisieren sich diese in jeder Person in Form von Meanings. Analog zu dieser semantischen Differenzierung gilt die Unterscheidung zwischen überindividuellem Sinn (Sinnkategorien) und individuellen Bedeutungen (Lebensbedeutungen).

Die Medizin und die Psychologie konkretisieren die philosophische Frage nach dem „Sinn des Lebens“, die nicht allge- mein beantwortet werden kann, auf individueller Ebene durch die Frage nach Lebensbedeutungen, die sowohl Orientierung als auch personale Bedeutungen meinen (Jankélévich 2017, S. 230). In diesem Sinne wurden, v.a. im angelsächsischen Raum, verschiedene Skalen zur Erfassung von Haltungen („attitudes“), Kohärenzgefühl („sense of coherence“) und Lebensbedeutungen („meaning in life") entwickelt (Valdés-Stauber et al. 2018). Im deutschsprachigen Raum haben Schnell und Becker (2007) den Fragebogen zu Lebensbedeutungen und Lebenssinn entwickelt und validiert; neben verschiedenen einzelnen, empirisch relevanten Lebensbedeutungen findet sich der abstrakte „Lebenssinn“ als erfahrbar durch „Sinnerfüllung“ und „Sinnkrise“ (Schnell 2009).

Das „Life Attitude Profile“ von Reker wurde nach psychometrischen Untersuchungen 1992 in revidierter Version (LAP-R) veröffentlicht (Reker und Peacock 1981; Reker 1992); die 6 Skalen zeigen eine gute interne Konsistenz (Cronbachs a 0,79-0,86) und eine gute Test-Retest-Reliabilität (0,77-0,87; Reker 1992). „Sinn“ wird in diesem Fragebogen durch 6 Dimensionen abgebildet, die auf Frankl $(1976,1994)$ rekurrieren und v. a. die Suche nach Ordnung, Kohärenz, Zielen und Erfüllung widerspiegeln (Reker 2000). Jede der 6 Dimensionen (Lebensziele, Kohärenz, Selbstwirksamkeit, Akzeptanz des Todes, existenzielle Leere und Suche nach Zielen) besteht aus 8 Fragen, 
Akronym Skalenbeschreibung

\section{Klinische Skalen}

ICD-Symptom-Rating

ISR

Hamburger Module zur Erfassung allgemeiner Aspekte psychosozialer Gesundheit für die therapeutische Praxis

Fragebogen zur Lebenszufriedenheit

(Modul Allgemeine Lebenszufriedenheit)

Globale Verlaufseinschätzung

Veränderung durch die Therapie

Helping Alliance Questionnaire

Lebenseinstellungen

Lebensziele

Kohärenz

Selbstwirksamkeit/

Verantwortungsbereitschaft

Akzeptanz des Todes DA

Existenzielle Leere $\quad$ EV

Suche nach Zielen GS

Personal meaning index PMI

Existential transcendence $\quad \mathrm{ET}$
Psychische Symptombelastung. Selbsteinschätzung der Schwere der psychischen Symptomatik in den vergangenen 2 Wochen (Angst, Zwang, Depression, Somatisierung, Essstörung, Zusatzskala), bestehend aus 29 Items. Likert-Skala von 0 bis 4 . Summenscore in einem Range von 0 bis max. 116. Wird v. a. in stationärer Psychotherapie verwendet

HEALTH-49 Selbstwirksamkeit. Selbstbeurteilungsinstrument zur Erfassung der psychosozialen Gesundheit. Für die vorliegende Untersuchung wird die Selbstwirksamkeit (Modul D) gewählt. Dieses Modul besteht aus 5 Items mit jeweils einer Antwortskala von 0 (stimmt nicht) bis 4 (stimmt sehr), sodass der Summenscore Werte zwischen 0 und 20 einnehmen kann

FLZ ${ }^{\mathrm{M}} \quad$ Lebenszufriedenheit. Selbstbeurteilungsinstrument der allgemeinen Lebenszufriedenheit. 8 Items. Antwortskala 1 (sehr zufrieden) bis 7 (sehr unzufrieden). Zur besseren Übersicht in den Regressionsanalysen wurde die Skala umgepolt. Der Range des Summenscore nimmt Werte zwischen 0 und 56 an

(in Anleh- Ermittlung mithilfe eines Patientenfragebogens, bestehend aus 7 Fragen mit Summenwerten zwinung) Psy- schen 7 und 35. Aus Forschungszwecken umgepolt: 1 (deutlich verschlechtert) bis 5 (deutlich verBaDo bessert)

HAQ-D Fragebogen zur Evaluation der Behandlung, hier aus Sicht des Patienten (11 Fragen mit Antwortrange von -3 bis +3 ). Gesamtrange: -33 bis +33

LAP-R Life Attitude Profile-Revised (Reker 1992; Deutsche Version von Mehnert et al. 2007)

PU Richtungen und Lebensaufgaben, Gefühl der individuellen Bedeutsamkeit

CO Bewusstsein und Zustimmung zu sich selbst, zu den anderen, zum Leben

CR Gefühl der Verantwortung, Entscheidungsfähigkeit, Freiheit und Kontrolle

DA Tod als zum Leben gehörig, Abwesenheit von Furcht davor

Negative Skala: Mangel an Sinn, Zielen, Richtung, Interessen, Entscheidungen

Suche von Herausforderungen, die das Leben bereichern könnten

Individuelle Bedeutung: PU + CO

ET Existenzielle Transzendenz: $(\mathrm{PU}+\mathrm{CO}+\mathrm{CR}+\mathrm{DA})-(\mathrm{EU}+\mathrm{GS})$

FLZ ${ }^{M}$ Fragebogen zur Lebenszufriedenheit - Modul, ICD Internationale statistische Klassifikation der Krankheiten und verwandter Gesundheitsprobleme, Psy$B a D o$ Basisdokumentation in der Psychotherapie

die auf einer Likert-Skala von 1 (stimme überhaupt nicht $\mathrm{zu}$ ) bis 7 (stimme völlig $\mathrm{zu}$ ) beantwortet werden können, wobei 4 Skalen positiv und 2 (existenzielle Leere und Suche nach Zielen) negativ ausgerichtet sind (wobei "Suche nach Zielen“ je nach Lebnskontext und Lebensphase positiv oder negativ zu bewerten ist). In - Tab. 1 sind die einzelnen Dimensionen und übergeordneten Indizes kurz beschrieben. Diese Skala wurde 2007 von Mehnert et al. (2007) ins Deutsche übersetzt und validiert.

\section{Fragestellungen und Methode}

\section{Fragestellungen}

Die vorliegende Studie untersucht die psychometrischen Eigenschaften der deutschen revidierten Version des Life Attitude Profile von Reker sowie mögli- che Assoziationen mit klinischen Outcome-Variablen. Diese allgemeine Fragestellung wird in folgende Teilfragestellungen untergliedert:

1. Wie hoch ist die Reliabilität der deutschen Version des LAP-R im Sinne der internen Konsistenz bei der spezifischen Stichprobe?

2. Wie ist die Faktorenstruktur des Frageboges als reflektives Messmodell für die Zielstichprobe?

3. Inwieweit sind die einzelnen Dimensionen des LAP-R unabhängig und messen, bezogen auf Lebenssinn, unterschiedliche Facetten desselben Sachverhalts?

4. Ist konvergente Validität mit anderen validierten Skalen gegeben?

5. Korreliert das Ausmaß der Veränderung der Lebensziele mit dem Ausmaß der Veränderung der (klinischen) Ergebnisqualitätsvariablen?

\section{Stichprobenbeschreibung}

An einer psychosomatischen Klinik wurden 154 Patientinnen und Patienten untersucht; anteilig $75 \%$ Frauen, 25\% Männer. Der Altersdurchschnitt betrug 40 Jahre; es lebten $53 \%$ der Teilnehmenden aktuell in einer Partnerschaft, und $42 \%$ waren Eltern. Bezogen auf Bildung und Beruf hatten $37 \%$ einen Hauptschulabschluss, $26 \%$ Abitur, $56 \%$ waren berufstätig. Die Erstdiagnose lautete in $51 \%$ der Fälle „depressive Episode“ und in $42 \%$,rezidivierende depressive Störung". Bei $58 \%$ der Patientinnen und Patienten wurden 2 oder mehr Diagnosen psychischer Störungen gestellt. Die häufigsten Zweitdiagnosen waren posttraumatische Belastungsstörung (17\%), Persönlichkeitsstörung und Suchtstörung (jeweils 16\%), Angststörung und somatoforme Störung (jeweils 15\%) so- 
Psychotherapeut 2021 -66:147-155 https://doi.org/10.1007/s00278-020-00481-3

(c) Der/die Autor(en) 2020

\section{J. Valdés-Stauber $\cdot$ H. Kämmerle $\cdot$ S. Bachthaler}

\section{Psychometrische Eigenschaften des Life Attitude Profile - Revised. Prüfung der deutschen Version in einem stationär-psychosomatischen Setting}

\section{Zusammenfassung}

Hintergrund. Die allgemeine Frage nach dem "Sinn des Lebens" wird empirisch in Form von allgemeinen und personalen Lebensbedeutungen formuliert. In der Medizin erhalten Lebensbedeutungen eine relevante Tragweite bei einem Verlust von Sinn, der als krankheitsfördernd angesehen wird, v. a. aus psychosomatischer Perspektive. Fragestellung. Die vorliegende Studie untersucht die psychometrischen Eigenschaften der deutschsprachigen revidierten Version des Life Attitude Profile (LAP-R) von Reker für eine Stichprobe stationär-psychosomatischer Patienten sowie mögliche Assoziationen mit klinischen Outcome-Variablen.

Material und Methoden. Die Stichprobe ( $n=138$ ) von elektiv stationär-psychosomatisch behandelten Patienten wird zu den Zeitpunkten Aufnahme und Entlassung untersucht. Reliabilität im Sinne von interner Konsistenz wird mit Cronbachs a ausgewertet: Konstruktvalidität wird mit konfirmatorischer Faktorenanalyse und konvergente Validität anhand von Korrelationsmatrizen mit validierten klinischen Skalen erfasst. Ergebnisse. Die interne Konsistenz des LAP-R für die einzelnen Dimensionen zeigt Cronbachs- $a$-Werte zwischen 0,69 und 0,85 , für den Gesamttest beträgt Cronbachs a 0,92. Die konfirmatorische Faktorenanalyse zeigt einen guten Daten-Fit (,root mean square error of approximation" [RMSEA], $x^{2}$ /Freiheitsgrade [df]), aber einen zu geringen Modell-Fit (,Tucker-Lewis index" [TLI], ", comparative fit index" [CFI]). Die Korrelationen zwischen den Dimensionen des LAP-R sind meist moderat. Die Korrelation zwischen dem Ausmaß der klinischen Besserung und der Zunahme der
Lebensbedeutungen ist mäßig, aber mit der rückblickenden therapeutischen Beziehung hoch.

Schlussfolgerung. Die deutsche Version des LAP-R zeigt für die Stichprobe stationärpsychosomatisch behandelter Patienten gute interne Konsistenz, gute konvergente Validität jedoch uneinheitliche Konstruktvalidität. Die übergeordneten Indizes („personal meaning index" [PMI], "existential transcendence" [ET]) korrelieren signifikanter mit klinischen Variablen als die einzelnen Dimensionen.

\section{Schlüsselwörter}

Lebensbedeutungen · Stationäre Psychosomatik - Therapeutische Beziehung . Neurotizismus · Konfirmatorische Faktorenanalyse

\section{Psychometric properties of the life attitude profile-revised. Testing the German version in an inpatient psychosomatic setting}
Abstract
Background. The general question of the "meaning of life" is empirically formulated in the form of general significances and personal meanings. In medicine "meaning of life" is relevant in the case of loss of meaning, which is considered disease-promoting, especially from a psychosomatic perspective.
Objective. This study examined the psychometric characteristics of the German language revised version of Reker's life attitude profile (LAP-R) for a sample of inpatient psychosomatic patients as well as possible associations with clinical outcome variables.
Material and methods. The study sample ( $n=138)$ consisted of elective patients treated in a hospital ward for psychosomatics who were examined at the times of admission
and discharge. Reliability was investigated in terms of internal consistency with Cronbach's a. Construct validity was assessed with confirmatory factor analysis. Convergent validity was measured using correlation matrices with validated clinical scales. Results. The internal consistency of the LAP-R for the single dimensions showed Cronbach's $a$ values between 0.69 and 0.85 and for the total test a Cronbach's $a$ of 0.92 . The confirmatory factor analysis showed a good data fit (root mean square error of approximation, RMSEA, $X^{2} /$ degrees of freedom [df]) but insufficient model fit (Tucker-Lewis index, TLI, comparative fit index, CFI). The correlations among the dimensions of the LAP-R were mostly moderate. The correlation between the extent of clinical improvement

and the increase in life attitudes/meanings was moderate; however, the correlation between increase in life attitudes and the goodness of therapeutic alliance was high. Conclusion. The German version of the LAP-R showed good internal consistency, good convergent validity but nonuniform construct validity for the sample of inpatient psychosomatically treated patients. The superordinate indices (personal meaning index, PMI and existential transcendence, ET) correlated more significantly with clinical variables than the single dimensions.

Keywords

Sources of meaning · Inpatient psychosomatic treatment · Neuroticism - Therapeutic alliance . Confirmatory factor analysis wie phobische Störung mit $11 \%$. Die Diagnosen waren klinisch in Anlehnung an die Internationale statistische Klassifikation der Krankheiten und verwandter Gesundheitsprobleme, 10. Ausg. (ICD10), erhoben worden. Die Stichprobe wies erwartungsgemäß höhere Neurotizismuswerte als der Bevölkerungsdurchschnitt auf (Valdés-Stauber et al. 2019).
Die durchschnittliche Aufenthaltsdauer in der Klinik betrug 44,3 Tage.

\section{Studiendesign}

Die Studie war prospektiv-naturalistisch angelegt und beinhaltete 2 Messzeitpunkte: die stationäre Aufnahme und den Entlasszeitpunkt. Die Aufnahme der Patien- tinnen und Patienten erfolgte indikativ in der abteilungseigenen Ambulanz. Die Daten wurden durch eine geschulte Psychologin erhoben, die nicht an der Behandlung der Patientinnen und Patienten beteiligt war.

In - Abb. 1 sind die progressiven Schwundraten dargestellt. Von 200 konsekutiv befragten und aufgeklärten Pati- 


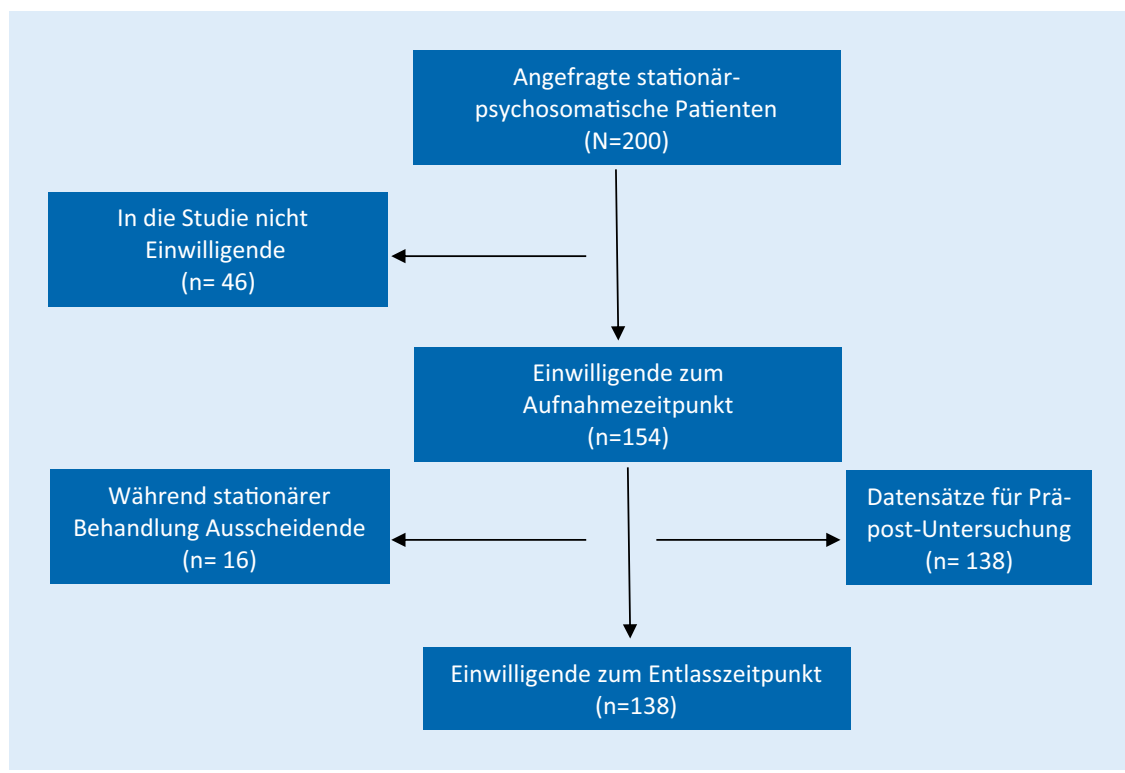

Abb. 1 ॥ Flussdiagramm zur einwilligenden Stichprobe hinsichtlich verwertbarer Datensätze für eine Prä-post-Untersuchung

entinnen und Patienten lehnten 46 Personen (23\% der Stichprobe) die Teilnahme ab. Von den verbliebenen 154 Einwilligenden nahmen 16 Personen am Ende der Behandlung (2. Messzeitpunkt) nicht mehr an der Untersuchung teil (Schwundrate von $8 \%$ ), sodass für 138 Personen Datensätze zu beiden Messzeitpunkten vorlagen.

Die klinischen und soziodemografischen Daten entstammen der qualitätssichernden Basisdokumentation. Folgende Variablen wurden anhand von vorwiegend validierten Skalen untersucht (zur Beschreibung: - Tab. 1):

a) klinische Variablen: psychische Symptombelastung, erfasst mithilfe des ICD-Symptom-Rating (ISR; Institut für Qualitätsentwicklung in der Psychotherapie und Psychosomatik 2006); Lebenszufriedenheit, erfasst mithilfe des Fragebogen zur Lebenszufriedenheit (FLZ ${ }^{\mathrm{M}}$; Henrich und Herschbach 2000); Selbstwirksamkeitsüberzeugung, erfasst mit dem Modul D der Hamburger Module zur Erfassung allgemeiner Aspekte psychosozialer Gesundheit für die therapeutische Praxis (HEALTH-49; Rabung et al. 2009);

b) subjektive Einschätzung der stationären Behandlung: Qualität der therapeutischen Beziehung, gemessen mit dem Patientenbogen des Helping
Alliance Questionnaire (HAQ-D; der globalen Veränderung durch die Therapie (7 Fragen, Likert-Skala 1-5, Werterange: 7-35), basierend auf der Basisdokumentation in der Psychotherapie (Psy-BaDo; Heuft und Senf 1998);

c) sinnbasierte Lebensbedeutungen: Hauptinstrument ist die deutsche Version des Life Attitude Profile Revised (LAP-R) zur Erfassung der Sinn- und Lebenseinstellungen, bestehend aus 48 Items, verteilt auf 6 Skalen, unterteilt in 4 positive und 2 negative Skalen (Reker 1992; Mehnert et al. 2007); hinzu kommen 2 übergeordnete Indizes: a) „personal meaning index“ (PMI): „individuelle Bedeutung“ aus der Summe „Lebensziele und Kohärenz“; b) „existential transcendence“ (ET): ,existenzielle Transzendenz" ([Lebensziele + Kohärenz + Selbstwirksamkeit/ Verantwortungsbereitschaft + Akzeptanz des Todes] - [existenzielle Leere + Suche nach Zielen]; Reker 2000; Anagnostopoulos et al. 2011). Die in dieser Studie verwendete deutsche Adaptation des LAP-R wurde von Mehnert et al. (2007) bei einer Stichprobe von 1083 Brustkrebspatientinnen eingesetzt. Die dort erhobenen Testgütekriterien weisen auf die Bassler et al. 1995); Einschätzung
Brauchbarkeit des Verfahrens in der medizinpsychologischen Forschung hin. Die vorliegende Untersuchung strebt an, diese Validierung für eine Stichprobe von stationär-psychosomatischen Patienten zu replizieren.

\section{Statistik}

Zur Prüfung der Normalitätsverteilung wurden die metrischen Variablen einem Shapiro-Wilk-Test unterzogen. Normal verteilt sind Dimensionen der Lebensbedeutungen (LAP-R), Lebenszufriedenheit $\left(\mathrm{FLZ}^{\mathrm{M}}\right)$, Selbstwirksamkeit (HEALTH-49), psychische Symptombelastung (ISR) und Veränderung durch die Therapie, nicht aber Einschätzung der Qualität der therapeutischen Beziehung.

Die interne Konsistenzen innerhalb der jeweiligen Dimensionen und für den Gesamttest wurden anhand von 2 Parametern untersucht: a) „average interitem covariance" (AIC): Korrelation der Fragen untereinander (Werte $\geq 0,30$ weisen auf eine gute Korrelation der Fragen untereinander hin); b) Cronbachs $\alpha$ : Maß der internen Konsistenz eines Tests, d.h., wie stark die Fragen einer Skala miteinander in Beziehung stehen (ein Wert $\geq 0,70$ für die gesamte Skala deutet auf eine gute interne Konsistenz hin).

Die konfirmatorische Faktorenanalyse (CFA) operiert mit latenten Variablen. Im vorliegenden Fall handelt es sich um die einzelnen Faktoren und um den Test als Ganzes. Bei der CFA soll unterschieden werden zwischen formativen und reflexiven Messmodellen (Zinnbauer und Eberl 2004): Bei reflexiven Messmodellen wird davon ausgegangen, dass die Indikatoren, die einer latenten Variable zugeordnet sind, von der latenten Variable kausal beeinflusst werden und somit auch die exogenen Variablen indirekt Einfluss auf die reflexiven Indikatoren haben, die in solchen Modellen austauschbar wären (Bollen 1989; Edwards und Bagozzi 2000). Für formative Messmodelle gilt hingegen: Die formativen Indikatoren begründen die latenten Variablen, sind kausal für deren Erklärung heranzuziehen; die formativen Indikatoren sind nicht austauschbar. Im vorliegenden Fall handelt es sich demnach um ein reflexives Messmodell. Folgende Gütemaße wer- 
Tab. 2 Reliabilität (interne Konsistenz nach Cronbachs $\alpha$ ) der deutschen Version des Life Attitude Profile - Revised bei einer Stichprobe (Messzeitpunkt post; $n=138$ ) stationär-psychosomatischer Patienten einer akut-psychosomatischen Krankenhausabteilung

\begin{tabular}{|c|c|c|c|}
\hline Einzelne Dimensionen (dazugehörige Items) & $M( \pm S D)$ & AIC & $\begin{array}{l}\text { Cronbachs } \\
a\end{array}$ \\
\hline 1. Lebensziele/Bestimmung $(1,2,5,18,26,31,37,48)$ & $3,82( \pm 1,18)$ & 1,14 & 0,85 \\
\hline 2. Kohärenz $(7,12,16,27,29,35,38,46)$ & $3,73( \pm 1,08)$ & 0,99 & 0,80 \\
\hline $\begin{array}{l}\text { 3. Selbstwirksamkeit/Verantwortungsbereitschaft }(3,11,17 \text {, } \\
19,23,30,39,45)\end{array}$ & $4,32( \pm 0,97)$ & 0,82 & 0,81 \\
\hline 4. Akzeptanz des Todes $(8,15,22,25,28,32,44,47)$ & $4,29( \pm 1,18)$ & 1,05 & 0,82 \\
\hline $\begin{array}{l}\text { 5. Existenzielle Leere } \\
(4,6,9,13,20,33,40,42)\end{array}$ & $4,17( \pm 0,83)$ & 0,52 & 0,66 \\
\hline$\rightarrow$ nach Tilgung der Items 4, 33 & - & 0,74 & 0,69 \\
\hline 6. Suche nach Zielen $(10,14,21,24,34,36,41,43)$ & $4,42( \pm 0,97)$ & 0,67 & 0,74 \\
\hline Gesamttest & - & 0,55 & 0,92 \\
\hline
\end{tabular}

Tab. 3 Prüfung der Konstruktvalidität anhand einer konfirmatorischen Faktorenanalyse der deutschen Version des Life Attitude Profile - Revised bei einer Stichprobe (Messzeitpunkt post; $n=138)$ stationär-psychosomatischer Patienten einer akut-psychosomatischen Krankenhausabteilung

\begin{tabular}{lll}
\hline & $\begin{array}{l}\text { Komplettes } \\
\text { Modell }\end{array}$ & $\begin{array}{l}\text { Reduziertes Modell } \\
\text { (Items: 4, 20, 21, 33, 35) }\end{array}$ \\
\hline Freie Parameter & 150 & 135 \\
\hline „Akaike information criterion“ & 50320 & 44793 \\
\hline "Bayesian information criterion“ & 50874 & 45292 \\
\hline Chi-Quadrat ( ${ }^{2}$ ) - Freiheitsgrade (df) & $2687 / 1074$ & $2062 / 854$ \\
\hline$X^{2}:$ df & 2,50 & 2,41 \\
\hline $\begin{array}{l}\text { Comparative fit index (CFI) } \\
\text { Tucker-Lewis index (TLI) }\end{array}$ & 0,72 & 0,77 \\
\hline $\begin{array}{l}\text { Root mean square error of approximati- } \\
\text { on (RMSEA) }\end{array}$ & 0,70 & 0,76 \\
\hline $\begin{array}{l}\text { Standardized root mean square residual } \\
\text { (SRMSR) }\end{array}$ & 0,094 & 0,069 \\
\hline
\end{tabular}

den berücksichtigt: a) $\chi^{2} /$ Freiheitsgrade $(d f)$ : Werte $<3,0$ werden als akzeptabel betrachtet; b) Gruppe der, „incremental fit indices" (IFI): Dazu gehören der „Tucker-Lewis index" (TLI) und der „,comparative fit index" (CFI). Sie vergleichen die Anpassung (Fit) des Zielmodells mit dem Fit des Baseline-Modells und geben an, um wie viel das Zielmodell besser auf die Daten passt als das Unabhängigkeitsmodell (die Werte variieren zwischen 0 und 1; die Mindestgrenze für einen guten Fit beträgt 0,90$)$; c) informationstheoretische Maße (AIC und BIC): Sie vergleichen Modelle nach unterschiedlichen Restriktionen. Das Modell mit dem niedrigeren Wert wird ausgewählt; d) approximative Datenanpassung (Daten-Fit): wird mit „root mean square error of approximation“ (RMSEA) gemessen. Für ein akzep- tables Modell soll der Wert $\leq 0,08$ betragen. Ergänzend kann das standardisierte Maß zur Gesamtbeurteilung der Residuen hinzugenommen werden („standardized root mean square residual", SRMR; günstig $<0,05$; Zinnbauer und Eberl 2004;

Die konvergente Validität wurde mit validierten Skalen geprüft, die tendenziell ähnliche Sachverhalte messen: Hierzu wurden Lebenszufriedenheit und Selbstwirksamkeitsüberzeugung als potenziell positiv und psychische Symptombelastung als potenziell negativ assoziiert ausgewählt.

Der Vergleich der Dimensionen des LAP-R untereinander erfolgte anhand einzigen Zeitpunkt (Entlassung), da ansonsten die Ergebnisse verzerrt worden Schreiber et al. 2006). von Korrelationsmatrizen $\mathrm{zu}$ einem wären. Pearson-Korrelationsmatrizen wurden zudem eingesetzt, um das Ausmaß der Korrelationen zwischen LAP-RDimensionen und klinischen Variablen darzustellen, und zwar einerseits im Querschnitt zum Aufnahmezeitpunkt (um die Bedeutung der Psychopathologie hervorzuheben), andererseits anhand der Korrelationen zwischen dem Ausmaß der Veränderung der Dimensionen und der klinischen Outcome-Variablen.

Alle statistischen Berechnungen wurden mit StataMP 13 und mit Mplus 7 durchgeführt.

\section{Ergebnisse}

Die interne Konsistenz der einzelnen Dimensionen des LAP-R für die Stichprobe weist bis auf eine Dimension hohe AIC-Werte $>0,30$ und Werte für Cronbachs $\alpha$ um meist 0,80 auf. Ausnahme ist die Dimension „existenzielle Leere“, wobei Cronbachs $\alpha$ nach Entfernung der Items 4 und 33 (Cronbachs $\alpha$ des Item jeweils höher als der Dimension) mit 0,69 im akzeptablen Bereich liegt. Besonders hoch ist die interne Konsistenz für den Gesamttest mit $\mathrm{AIC}=0,55$ und Cronbachs $\alpha=0,92$ ( $\bullet$ Tab. 2).

Alle standardisierten Ladungen der Einzelitems auf die jeweiligen latenten Variablen 1. Ordnung (die 6 Dimensionen) und auf die latente Variable 2. Ordnung (der Test insgesamt) sind in $\bullet$ Abb. 2 dargestellt.

Die Gütemaße fallen unterschiedlich aus: Während $\chi^{2} / \mathrm{df}$ mit 2,5 (also: $<3,0$ ) und RMSEA mit 0,07 (also: $<0,08$ ) für einen guten Modell-Fit sprechen, sind die Werte für die Incremented fit indices (CFI und TLI) mit etwa 0,73 zu niedrig (also: $<0,90)$. Werden die Items mit standardisierten Ladungen, die unterhalb von 0,30 liegen, entfernt, dann verbessern sich alle Parameter (CFI und TLI), bleiben aber noch zu niedrig (•Tab. 3). Auf den Gesamttest laden alle Dimensionen signifikant mit standardisierten Ladungen $>0,30$ (•Tab. 3 ).

Erwartungsgemäß korrelieren alle Dimensionen im Querschnitt (hier zum Entlasszeitpunkt) signifikant untereinander, da sie alle Lebenseinstellungen betreffen; die Dimension „existenzielle Leere"korreliert negativ mit den anderen Di- 


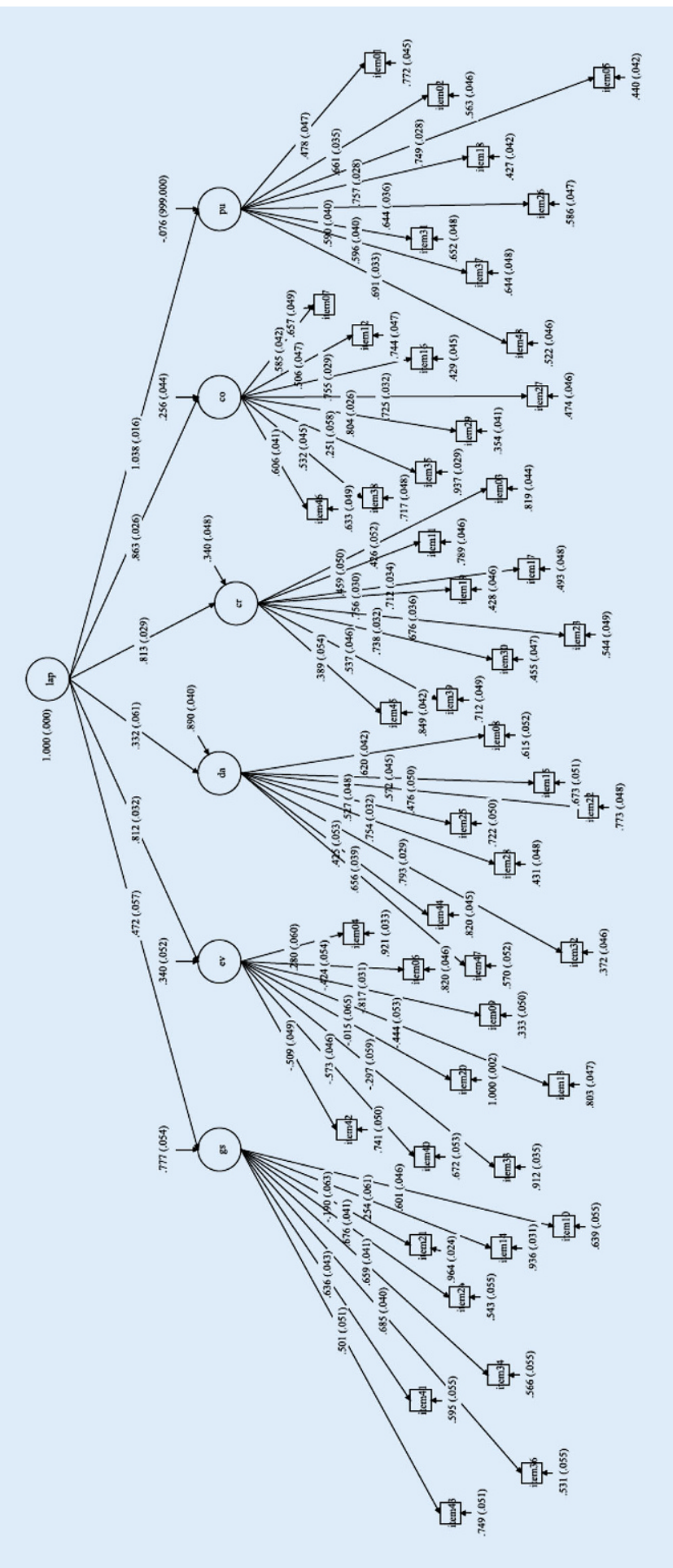

Die Prä-post-Veränderungen für alle Dimensionen des LAP-R (außer „existenzielle Leere“) und für die übergeordneten Indizes (PMI und ET) korrelieren signifikant positiv mit der von den Patienten eingeschätzten Güte der therapeutischen Beziehung $(r=0,19-0,47)$ : Je positiver die Beziehung eingeschätzt wird, desto deutlicher sind die Veränderungen auf dem LAP-R in die zu erwartenden Richtung, insbesondere für Selbstwirksamkeit $(r=0,42)$. Bezogen auf die LAP-R-Dimensionen, nur das Ausmaß der Veränderung von "Lebensziele“ $(\mathrm{r}=0,21-0,29)$ und die übergeordneten Indizes $(r=0,19-0,48)$ weisen statistisch signifikante positive Assoziationen mit den 5 hier untersuchten Outcome-Variablen (•Tab. 6).

Abb. $2<$ Konfirmatorische Faktorenanalyse zur Prüfung der Konstruktvalidität auf Basis eines reflektiven Messmodells. Die 6 einzelnen Dimensionen werden als latente Variablen 1. Ordnung und der Gesamttest als latente Variable 2. Ordnung behandelt. Auf den Verbindungslinien stehen die standardisierten Faktorenladungen; unterhalb der einzelnen Items stehen die Fehlervariablen für die Indikatoren. Iap Life Attitude Profile, gs Suche nach Zielen, ev existenzielle Leere, da Akzeptanz des Todes, $\mathrm{cr}$ Selbstwirksamkeit/ Verantwortungsbereitschaft, co Kohärenz, pu Lebensziele mensionen, da sie eine umgekehrte Bewertungsrichtung hat. Die übergeordneten Indizes als Verdichtung der Dimensionen korrelieren besonders hoch mit den einzelnen Dimensionen ( $r=0,27$ bis 0,92, meist aber >0,50; • Tab. 4).

Erwartungsgemäß korrelieren die Dimensionen und Indizes von LAP-R im Querschnitt zum Aufnahmezeitpunkt positiv mit Lebensqualität $(r=0,22-0,65)$ und Selbstwirksamkeit $(r=0,23-0,61)$ sowie negativ mit dem Ausmaß der psychischen Belastung $(\mathrm{r}=-0,34$ bis $-0,47)$; für die Dimension „existenzielle Leere“ als negative Eigenschaft verhält es sich umgekehrt (•Tab. 5).

\section{Diskussion}

Die vorliegende Untersuchung befasst sich mit den psychometrischen Eigenschaften der deutschen Version des LAP-R für eine Stichprobe stationär behandelter Patienten in einer psychosomatischen Abteilung eines Allgemeinkrankenhauses. Untersucht werden die interne Konsistenz, die Konstruktvalidität und die konvergente Validität. Eine exploratorische Faktorenanalyse ist im vorliegenden Fall nicht erforderlich, da die Faktoren aus der Theorie bereits vorgegeben sind und nicht explorativ untersucht werden müssen. Allerdings werden in der deutschen Fassung von Mehnert et al. (2007) 5 Faktoren, in der türkischen Fassung von Erci (2008) 4 und in der griechischen von Anagnostopoulos et al. (2011) ebenfalls 4 Faktoren extrahiert. Eine gute Reliabilität im Sinne einer internen Konsistenz kann in der vorliegenden Stichprobe bestätigt werden; Cronbachs a beträgt zwischen 0,80 und 0,85 , ähnlich wie in der kanadischen Stichprobe von Reker (1992) und in der Stichprobe von Mehnert et al. (2007). Dagegen sind für die vorliegende Stichprobe die Cronbachs- $\alpha$-Werte für „existenzielle Leere“ $(0,69)$ und „Suche nach Zielen“ $(0,74)$ etwas niedriger als in den genannten Studien, die andere Stichproben zugrunde legten.

Die konfirmatorische Faktorenanalyse zeigt ein gemischtes Bild, denn, wäh- 


\begin{tabular}{|c|c|c|c|c|c|c|c|}
\hline & Lebensziele & Kohärenz & $\begin{array}{l}\text { Selbstwirksam- } \\
\text { keit }\end{array}$ & $\begin{array}{l}\text { Akzeptanz des } \\
\text { Todes }\end{array}$ & $\begin{array}{l}\text { Existenzielle } \\
\text { Leere }\end{array}$ & $\begin{array}{l}\text { Suche nach } \\
\text { Lebenszielen }\end{array}$ & LAP-PMI \\
\hline & $\mathbf{r}^{p}$ & $r^{p}$ & $\mathbf{r}^{p}$ & $\mathbf{r}^{p}$ & $\mathbf{r}^{p}$ & $\mathbf{r}^{p}$ & $\mathbf{r}^{p}$ \\
\hline Lebensziele & - & - & - & - & - & - & - \\
\hline Kohärenz & $0,77^{* * *}$ & - & - & - & - & - & - \\
\hline $\begin{array}{l}\text { Selbstwirksamkeit/ } \\
\text { Verantwortungsbereitschaft }\end{array}$ & $0,64^{* * *}$ & $0,53^{* * *}$ & - & - & - & - & - \\
\hline Akzeptanz des Todes & $0,29 * * *$ & $0,35^{* * *}$ & $0,34^{* * *}$ & - & - & - & - \\
\hline Existenzielle Leere & $-0,45^{* * *}$ & $-0,34^{* * *}$ & $-0,17^{*}$ & n.s. & - & - & - \\
\hline Suche nach Zielen & $0,20^{*}$ & $0,34^{* * *}$ & $0,22^{* *}$ & $0,22^{* *}$ & $0,29^{* * *}$ & - & - \\
\hline LAP - PMI & $0,89 * * *$ & $0,92^{* * *}$ & $0,59 * * *$ & $0,31^{* *}$ & $-0,48^{* * *}$ & $0,27^{* *}$ & - \\
\hline LAP - ET & $0,83^{* * *}$ & $0,78^{* * *}$ & $0,70^{* * *}$ & $0,54^{* * *}$ & $-0,66^{* * *}$ & n.s. & $0,85^{* * *}$ \\
\hline \multicolumn{8}{|c|}{$\begin{array}{l}r \text { Korrelationskoeffizient, } p \text { Signifikanzniveau des Tests, PMI Personal meaning index, ET Existential transcendence, } n \text {. s. Korrelationstest statistisch nicht signifi- } \\
\text { kant } \\
*<0,05 \\
* *<0,01 \\
* * *<0,001\end{array}$} \\
\hline
\end{tabular}

Tab. 5 Prüfung der Konvergenzvalidität des Life Attitude Profile (LAP) - Revised (LAP-R) mit Outcome-Variablen (Messzeitpunkt prä; $n=154$ )

\begin{tabular}{|c|c|c|c|}
\hline \multicolumn{4}{|c|}{ Korrelation zwischen Dimensionen LAP-R und klinischen Variablen } \\
\hline & $\begin{array}{l}\text { Lebenszufrieden- } \\
\text { heit }\left(F L Z^{M}\right)\end{array}$ & $\begin{array}{l}\text { Psychische Belas- } \\
\text { tung (ISR) }\end{array}$ & $\begin{array}{l}\text { Selbstwirksamkeit } \\
\text { (HEALTH-49) }\end{array}$ \\
\hline & $\mathbf{r}^{p}$ & $\mathbf{r}^{p}$ & $\mathbf{r}^{p}$ \\
\hline Lebensziele & $0,65^{* * *}$ & $-0,42^{* * *}$ & $0,61^{* * *}$ \\
\hline Kohärenz & $0,39 * * *$ & $-0,34^{* * *}$ & $0,54^{* * *}$ \\
\hline $\begin{array}{l}\text { Selbstwirksamkeit/ } \\
\text { Verantwortungsbereitschaft }\end{array}$ & $0,59 * * *$ & $-0,43^{* * *}$ & $0,51^{* * *}$ \\
\hline Akzeptanz des Todes & $0,22^{*}$ & $-0,36^{* * *}$ & $0,23^{* *}$ \\
\hline Existenzielle Leere & $-0,37^{* * *}$ & $0,32^{* * *}$ & $-0,35^{* * *}$ \\
\hline Suche nach Zielen & $0,22^{*}$ & n.s. & $0,25^{* *}$ \\
\hline LAP-PMI & $0,51^{* * *}$ & $-0,37^{* * *}$ & $0,57^{* * *}$ \\
\hline LAP-ET & $0,55^{* * *}$ & $-0,47^{* * *}$ & $0,55^{* * *}$ \\
\hline \multicolumn{4}{|c|}{$\begin{array}{l}r \text { Korrelationskoeffizient, } p \text { Signifikanzniveau des Tests, } P M I \text { Personal meaning index, ET Existential tran- } \\
\text { scendence, } \Delta \text { Differenz Aufnahme-Entlassung, }{ }^{*}<0,05, * *<0,01,{ }^{* *}<0,001, n \text {. } \text {. } \text {. nicht signifikant, } \\
\text { FLZ }{ }^{M} \text { Fragebogen zur Lebenszufriedenheit, ISR ICD-Symptom-Rating, HEALTH-49 Hamburger Module } \\
\text { zur Erfassung allgemeiner Aspekte psychosozialer Gesundheit für die therapeutische Praxis. Darstellung } \\
\text { Korrelationen: Zur besseren Übersicht sind signifikante positive Korrelationen nach links und signifikan- } \\
\text { te negative nach rechts in den Zellen verschoben worden }\end{array}$} \\
\hline
\end{tabular}

rend $\chi^{2} / \mathrm{df}$ und RMSEA einen guten Fit aufweisen, sind die Incremental Fit Indices nicht ausreichend, auch nicht nach Bereinigung um 5 Items. Ein Grund dafür dürfte in der knappen Stichprobe liegen, wenn man dieZahl der Dimensionen und Items pro Dimension berücksichtigt, wie es die psychometrische Forschung nahelegt (Gagné und Hankock 2006; Şahin und Anil 2017). Vergleiche zwischen dem CFI von Bentler und Bonett sowie dem RMSEA von Steiger und Lind zeigen, dass Ersterer eher für explorative und Letzterer für konfirmatorische Kontexte geeignet sind, da sie von unterschiedli- chen Baseline-Modellen ausgehen (Rigdon 1996). Außerdem handelt es sich hier um ein Modell 2. Ordnung, d.h., es werden 2 Ebenen von latenten Variablen zugrunde gelegt. Anhand der standardisierten Ladungen könnte der Fragebogen um die Items mit niedrigeren oder nicht statistisch signifikanten Ladungen reduziert werden, da es sich um ein reflektives Messmodell handelt (Zinnbauer und Eberl 2004).

Zur Beantwortung der 3. Fragestellung, inwieweit die einzelnen Dimensionen des LAP-R unabhängig sind und, bezogen auf den Lebenssinn, unterschied- liche Facetten desselben Sachverhaltes messen, wurden Korrelationsmatrizen zwischen den Dimensionen des LAP und Outcome-Variablen (Lebenszufriedenheit, allgemeine psychische Belastung und Selbstwirksamkeitsüberzeugung) durchgeführt. Die Ergebnisse weisen darauf hin, dass von einer ausreichenden konvergenten Validität ausgegangen werden kann, da erwartete signifikante positive bzw. negative Korrelationen mit Dimensionen des LAP-R, die ebenfalls personale Ressourcen messen, ermittelt werden konnten.

Die Tatsache, dass alle Dimensionen miteinander korrelieren, die Koeffizienten jedoch selten über $r=0,50$ betragen, spricht dafür, dass die Dimensionen Ähnliches messen, aber nicht deckungsgleich sind; so behalten jede Dimension und der Test als Ganzes ihre Berechtigung. Von besonderer Bedeutung scheinen die übergeordneten Indizes zu sein: Der PMI und die ET korrelieren stark mit den einzelnen Dimensionen und den OutcomeVariablen. In der weiteren klinischen Forschung könnte das 6-dimensionale Modell vereinfachend auf diese 2 Indizes reduziert werden.

Die vorliegende Untersuchung weist auf eine ausreichende interne Konsistenz sowie gute konvergente Validität und teilweise akzeptable Konstruktvalidität der deutschen Version des LAP-R für die untersuchte Stichprobe der indikativ stationär-psychosomatisch be- 
Tab. 6 Korrelation zwischen Ausmaß der Veränderung der Dimensionen des Life Attitude Profile - Revised $(L A P-R ; \Delta)$ und Ausmaß der Besserung der klinischen Zielvariablen $(\Delta)$ durch stationäre Behandlung $(n=138)$

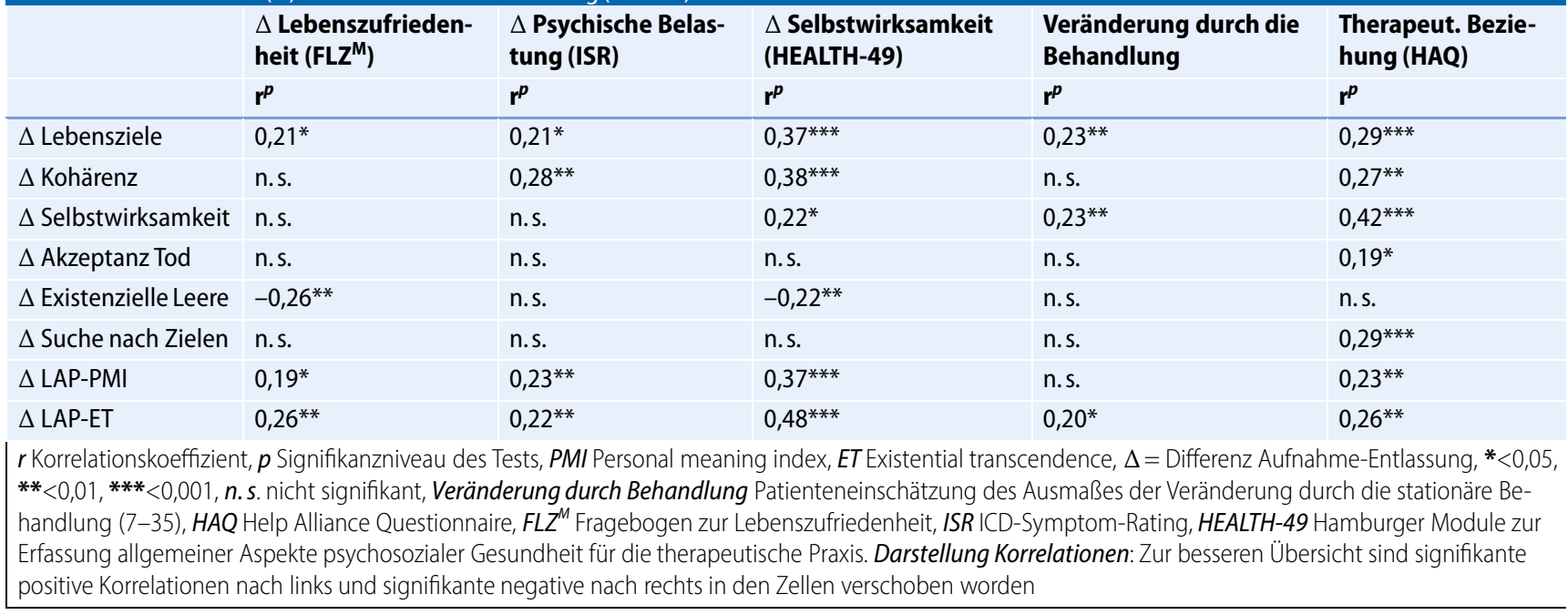

handelten Patienten hin. Wie bereits in einer anderen Arbeit festgestellt, kann von gemischten robusten und variablen wertebasierten Anteilen der Persönlichkeit ausgegangen werden, die nicht deckungsgleich mit Persönlichkeitsdimensionen sind (Valdés-Stauber et al. 2019). Die hohe Korrelation mit der Qualität der therapeutischen Beziehung könnte ein Hinweis für eine Bereitschaft zur Konstruktion von Sinn durch Therapie und zur Bildung von interpersonellen Begegnungsräumen sein, die von Vertrauen und Zuversicht zeugen, ähnlich wie es indikationsstellende Vorgespräche in der Psychotherapie leisten können (Bachthaler und Valdés-Stauber 2017).

\section{Limitationen der Studie}

Die relevanteste Limitation der Studie ist die fehlende Vergleichbarkeit mit einer Stichprobe aus der Allgemeinbevölkerung, die Aufschluss darüber geben könnte, inwiefern Lebenseinstellungen robust und von der klinisch relevanten psychischen Verfassung unabhängig sind. Die Prä-post-Untersuchungen zur Ermittlung der Veränderung der Lebenseinstellungen sind am ehesten als Merkmalsveränderungen während der Behandlung zu verstehen (ValdésStauber et al. 2020), allerdings können Messfehler, die kaum von realen Merkmalsveränderungen $\mathrm{zu}$ unterscheiden wären, einen Einfluss auf die gemesse- nen Unterschiede haben. Diese wissenschaftstheoretische Unsicherheit wird verstärkt durch eine mögliche Fehlerkumulierung bei wiederholten Messungen (verzerrtes Verständnis der Fragen und auch Inflation des Cronbachs- $\alpha$-Werts im Sinne falsch-positiver Prä-post-Unterschiede). Eine weitere statistische Limitation ergibt sich daraus, dass die Prä-post-Unterschiede geringer sind, je höher der Ausgangswert war, weshalb die Assoziationen zwischen dem Ausmaß der Veränderung der Lebenseinstellungen und der klinischen Variablen verzerrt sein könnten. Eine zusätzliche Limitation der Studie ist die begrenzte Generalisierbarkeit der Ergebnisse, denn einerseits bezieht sich die Stichprobe ausschließlich auf eine Gruppe von Menschen mit psychischen Störungen, andererseits können unterschiedliche Patientengruppen (Herzchirurgie, Dermatologie etc.) aus differierenden krankheitsbedingten Daseinseinrichtungen abweichende Profile aufweisen, wie auch psychosomatische Abteilungen und Settings je nach Ausrichtung und Versorgungsphilosophie unterschiedliche Patientinnen und Patienten erreichen.

\section{Fazit für die Praxis}

\footnotetext{
- Die psychometrische Untersuchung der deutschen Version des LifeAttitude-Profile-Revised(LAP-R)-Fragebogens zeigt, bezogen auf eine
}

Gruppe stationär-psychosomatischer Patientinnen und Patienten, eine gute Durchführungs- und Auswertungsvalidität, gute Reliabilität und konvergente Validität. Die Befunde zur Konstruktvalidität entsprechen jedoch nicht gänzlich den theoretischen Erwartungen.

- In logotherapeutisch orientierten Settings könnte der Fragebogen LAP-R unter methodischen Gesichtspunkten sowohl bei der Eingangsdiagnostik als auch zur Dokumentation der Einstellungsveränderungen bei Patienten empfohlen werden.

- Besondere Aufmerksamkeit sollte Patienten mit höheren Werten bei der Dimension „existenzielle Leere" geschenkt werden, da sie eine geringere Lebenszufriedenheit, geringere Selbstwirksamkeit sowie niedrigere Werte für die Güte der therapeutischen Beziehung bei stärkerer psychischer Belastung aufweisen.

- Da es eine Wechselwirkung zwischen der Güte der therapeutischen Beziehung und dem Veränderungsgrad der sinnbasierten Dimensionen besteht, müsste im Verlauf der Therapie auf beide Aspekte geachtet werden. 


\section{Korrespondenzadresse}

Prof. Dr. Juan Valdés-Stauber

Zentrum für Psychiatrie Südwürttemberg

Ravensburg, Deutschland

juan.valdes-stauber@zfp-zentrum.de

Funding. Open Access funding enabled and organized by Projekt DEAL.

\section{Einhaltung ethischer Richtlinien}

Interessenkonflikt. J.Valdés-Stauber, H. Kämmerle und S. Bachthaler geben an, dass kein Interessenkonflikt besteht.

Alle beschriebenen Untersuchungen am Menschen oder an menschlichem Gewebe wurden im Einklang mit nationalem Recht sowie gemäß der Deklaration von Helsinki von 1975 (in der aktuellen, überarbeiteten Fassung) durchgeführt. Die Studie wurde von der Ethikkommission der Universität Ulm (Antrag Nr. 03/2018) genehmigt. Von allen beteiligten Patienten liegt eine Einverständniserklärung vor.

Open Access. Dieser Artikel wird unter der Creative Commons Namensnennung 4.0 International Lizenz veröffentlicht, welche die Nutzung, Vervielfältigung, Bearbeitung, Verbreitung und Wiedergabe in jeglichem Medium und Format erlaubt, sofern Sie den/die ursprünglichen Autor(en) und die Quelle ordnungsgemäß nennen, einen Link zur Creative Commons Lizenz beifügen und angeben, ob Änderungen vorgenommen wurden.

Die in diesem Artikel enthaltenen Bilder und sonstiges Drittmaterial unterliegen ebenfalls der genannten Creative Commons Lizenz, sofern sich aus der Abbildungslegende nichts anderes ergibt. Sofern das betreffende Material nicht unter der genannten Creative Commons Lizenz steht und die betreffende Handlung nicht nach gesetzlichen Vorschriften erlaubt ist, ist für die oben aufgeführten Weiterverwendungen des $\mathrm{Ma}$ terials die Einwilligung des jeweiligen Rechteinhabers einzuholen.

Weitere Details zur Lizenz entnehmen Sie bitte der Lizenzinformation auf http://creativecommons.org/ licenses/by/4.0/deed.de.

\section{Literatur}

Anagnostopoulos F, Slater J, Fitzsimmons D, Kolokotroni P (2011) Exploring global meaning in Greek breast cancer patients: validation of the Life Attitude Profile-Revised (LAP-R). Psychooncology 20:419-427

Bachthaler S, Valdés-Stauber J (2017) Indikationsstellende Vorgespräche in der stationären Psychosomatik: Wirksamkeit einer Mikrointervention auf Behandlungszuversicht. Psychotherapeut 62:361-370

Bassler M, Portratz B, Krauthauser H (1995) Der "Helping Alliance Questionaire" von Luborsky. Psychotherapeut 40:23-33

Bollen KA (1989) Structural equations with latent variables. Wiley, New York
Edwards JR, Bagozzi RP (2000) On the nature and direction of relationships between constructs and measures. Psychol Methods 5:155-174

Erci B (2008) Meaning in life for patients with cancer: validation of the Life Attitude Profile-Revised Scale. J Adv Nurs 62(6):704-711

Frankl V (1976) Der Mensch auf der Suche nach Sinn: Zur Rehumanisierung der Psychotherapie. Herder, Freiburg

Frankl V (1994) Ärztliche Seelsorge - Grundlagen der Logotherapie und Existenzanalyse. Fischer, Frankfurta.M.

Gagné $P$, Hankock GR (2006) Measurement model quality, sample size, and solution propriety in confirmatory factor models. Multivariate Behav Res 41:65-83

Heidegger M (2006) Sein und Zeit. 19. Aufl. Tübingen: Max Niemeyer. Ersterscheinung 1927

Henrich G, Herschbach P (2000) Questions on life satisfaction (FLZM) - a short questionnaire for assessing subjective quality of life. Eur J Psychol Assess 16:150-159

Heuft G, Senf W (Hrsg) (1998) Praxis der Qualitätssicherung in der Psychotherapie: Das Manual zur Psy-BaDo. Thieme, Stuttgart (Auch in: Institut für Qualitätssicherung in der Psychotherapie (QiP): Ergebnis-Dokumentation Patient $\mathrm{B}_{2}$ : https:// psybado.de/dokugalerie/patergb2.rtf)

InstitutfürQualitätsentwicklung in derPsychotherapie und Psychosomatik (2006) ICD-10 Symptom Rating (ISR). Qualitas, München

Jankélévitch V (2017) Der Tod. Suhrkamp, Frankfurt a. M.

Kant I (1999) Kritik der reinen Vernunft. Köln: Parkland. Basierend auf der 2. Aufl.von 1787

Mehnert A, Müller D, Koch U (2007) Die Erfassung von sinnbasierten Bewältigungsstrategien und Lebenseinstellungen. Die deutsche Adaptation des Life Attitude Profile-Revised (LAP-R) an einer repräsentativen Stichprobe und Brustkrebspatientinnen. Z Klin Psychol Psychother 36:176-188

Overmann U (2000) Die Methode der Fallrekonstruktion in der Grundlagenforschung sowie der klinischen und pädagogischen Praxis. In: Kraimer K (Hrsg) Die Fallrekonstruktion. Sinnverstehen in der sozialwissenschaftlichen Forschung. Suhrkamp, Frankfurt a.M., S 28-156

Rabung S, Harfst T, Kawski S, Koch U et al (2009) Psychometrische Überprüfung einer verkürzten Version der "Hamburger Module zur Erfassung allgemeiner Aspekte psychosozialer Gesundheit für die therapeutische Praxis" (HEALTH-49). ZPsychosom Med Psychother 55:162-179

Reker GT (1992) Manual of the life attitude profilerevised (LAP-R). Student Psychologist Press, Peterborough

Reker GT (2000) Theoretical perspective, dimensions, and measurement of existential meaning. In: Reker GT, Chamberlain K (Hrsg) Exploring existential meaning: optimizing human development across the life span. SAGE, Thousand Oaks, S39-55

Reker GT, Peacock ET (1981) The life attitude profile (LAP): a multidimensional instrument for assessing attitudes toward life. Can J Behav Sci 13:264-273

Rigdon EE (1996) CFI versus RMSEA: a comparison of two fit indexes for structural equation modeling. Struct Equ Modeling 3-4:369-379

Schnell T (2009) The sources of meaning in life questionnaire (SoMe): relations to demographics and well-being. JPosit Psychol 4:483-499
Schnell T, Becker P (2007) LeBe - Fragebogen zu Lebensbedeutungen und Lebenssinn. Hogrefe, Göttingen

Schreiber JB, Nora A, Stage FK, Barlow EA, King J (2006) Reporting structural equation modeling and confirmatory factor analysis results: a review. JEducRes 99:323-338

Valdés-Stauber J (2018) Verstehen in der Psychiatrie II: Vom Unverständlichen zur Verstehensdisposition. Nervenarzt 89:1032-1042

Valdés-Stauber J, Kämmerle H, Bachthaler S (2019) Veränderung von Lebensbedeutungen während stationär-psychosomatischer Behandlung (In review)

Valdés-Stauber J, Lemanczyk R, Kilian R (2018) Sources of meaning in family caregivers of terminally ill patients supported by a palliative nursing care team: A naturalistic three-month cohort study. Palliat Support Care 16:246-259

Valdés-Stauber J, Kämmerle H, Bachthaler S (2020) Change of life attitudes during inpatient psychosomatic treatment. J Humanist Psychol. (ImDruck)

Zinnbauer M, Eberl M (2004) Die Überprüfung von Spezifikation und Güte von Strukturgleichungsmodellen:Verfahren und Anwendungen. Schriften zur Empirischen Forschung und Quantitativen Unternehmensplanung, Bd. 21/2004 Ludwig-Maximilians-Universität, München

Şahin A, Anil D (2017) The effects of test length and sample size on item parameters in item response theory. Educ Sci Theory Pract 17:321-335 